2010-03-11

\title{
Characterization of Optical Constants for Uranium from 10 to 47 $\mathrm{nm}$
}

Nicole Brimhall

nikif@byu.net

Nicholas Herrick

David D. Allred

allred@byu.edu

R. Steven Turley

Michael Ware

See next page for additional authors

Follow this and additional works at: https://scholarsarchive.byu.edu/facpub

Part of the Astrophysics and Astronomy Commons, and the Physics Commons

\section{Original Publication Citation}

Nicole Brimhall, Nicholas Herrick, David D. Allred, R. Steven Turley, Michael Ware, and Justin Peatross, "Characterization of Optical Constants for Uranium from $1 \mathrm{~nm}$ to $47 \mathrm{~nm}$," Applied Optics Vol. 49, Iss. 9, pp. 1581-1585 (21).

\section{BYU ScholarsArchive Citation}

Brimhall, Nicole; Herrick, Nicholas; Allred, David D.; Turley, R. Steven; Ware, Michael; and Peatross, Justin, "Characterization of Optical Constants for Uranium from 10 to 47 nm" (2010). Faculty Publications. 841. https://scholarsarchive.byu.edu/facpub/841

This Peer-Reviewed Article is brought to you for free and open access by BYU ScholarsArchive. It has been accepted for inclusion in Faculty Publications by an authorized administrator of BYU ScholarsArchive. For more information, please contact ellen_amatangelo@byu.edu. 


\section{Authors}

Nicole Brimhall, Nicholas Herrick, David D. Allred, R. Steven Turley, Michael Ware, and Justin Peatross

This peer-reviewed article is available at BYU ScholarsArchive: https://scholarsarchive.byu.edu/facpub/841 


\title{
Characterization of optical constants for uranium from 10 to $47 \mathrm{~nm}$
}

\author{
Nicole Brimhall, Nicholas Herrick, David D. Allred, R. Steven Turley, \\ Michael Ware, and Justin Peatross* \\ Department of Physics and Astronomy, Brigham Young University, Provo, Utah 84602, USA \\ ${ }^{*}$ Corresponding author: peat@ byu.edu
}

Received 4 January 2010; accepted 5 February 2010;

posted 22 February 2010 (Doc. ID 122140); published 11 March 2010

\begin{abstract}
We use a laser high-harmonics-based extreme-ultraviolet (EUV) polarimeter to determine the optical constants of elemental uranium in the wavelength range from 10 to $47 \mathrm{~nm}$. The constants are extracted from the measured ratio of p-polarized to s-polarized reflectance from a thin uranium film deposited in situ. The film thickness is inferred from a spectroscopic ellipsometry measurement of the sample after complete oxidation in room air. Uranium has been used as a high-reflectance material in the EUV. However, difficulties with oxidation prevented its careful characterization previous to this study. We find that measured optical constants for uranium vary significantly from previous estimates. (C) 2010 Optical Society of America

OCIS codes: $\quad 160.3900,160.4760,120.4530,120.5410,310.6860,340.7480$.
\end{abstract}

\section{Introduction}

Multilayer mirrors in the extreme-ultraviolet (EUV) wavelength range $(10-100 \mathrm{~nm})$ are essential optical elements for applications such as lithography, astronomy, and high-resolution microscopy [1-3] . To design mirrors with optimal performance, the optical constants need to be measured for materials in the EUV. Unfortunately, the optical properties of relatively few materials are well-characterized in this wavelength range [4-6]. For example, we recently found that accepted optical constants for copper in the EUV were incorrect due to inadvertent sample oxidation [7]. A lack of experimental characterization is especially common for materials in the actinide series, where reactivity, toxicity, and (sometimes) radioactivity adds challenges to taking optical measurements.

Uranium has demonstrated good performance as a multilayer component in the EUV, but the literature contains little measured data for its optical properties. Faldt and Nisson [8] measured optical constants

0003-6935/10/091581-05\$15.00/0

(C) 2010 Optical Society of America of uranium evaporated in situ in the wavelength range from 50 to $2000 \mathrm{~nm}$, which overlaps only the long-wavelength portion of the EUV; they used ellipsometry and normal-incidence reflectometry. On the other hand, Cukier and co-workers [9] measured the photoabsorption of uranium films at the much shorter wavelengths of 2.7 to $9.5 \mathrm{~nm}$, where oxidation was prevented using aluminum capping layers. For a large range of wavelengths in the EUV, no data are available for elemental uranium.

Members of our group produced multilayer uranium-silicon mirrors for use on the Imager for Magnetopause-to-Aurora Global Exploration (IMAGE) satellite launched in 2000 [1], which required high reflectance at $30.4 \mathrm{~nm}$ but low reflectance at $58.4 \mathrm{~nm}$. These multilayers were designed using theoretical estimates for the optical constants of uranium at the design wavelengths [10-13]. However, the fabricated mirrors reflected differently from what was predicted, which raised questions about the accuracy of these theoretical values.

We present the measured optical constants of uranium in the range from 10 to $47 \mathrm{~nm}$ characterized using the high-harmonics-based polarimeter described in Ref. [14] and the polarization-ratio reflectance 
measurement technique described in Ref. [15]. To our knowledge, this is the first time the optical constants of elemental uranium have been measured in this wavelength range. We find that the measured constants differ significantly from theoretical values [10-13] referenced on the Center for X-Ray Optics ( $\overline{\mathrm{CX}} \overline{\mathrm{RO}})$ website [16]. The revised constants would have significantly aided in the design of the IMAGE mirror [1] and resolve discrepancies in the previous study between multilayer designs and the measured reflectance data [17].

\section{Deposition of Uranium and Measurements}

We took our measurements using an EUV polarimeter instrument based on laser-generated high harmonics. This instrument, which is described in Ref. [14], uses $800 \mathrm{~nm}, 10 \mathrm{~mJ}, 35 \mathrm{fs}$, and $10 \mathrm{~Hz}$ laser pulses focused into a noble gas to produce polarized and directional high-harmonic light in the EUV wavelength range. Many harmonic frequencies (with wavelengths distributed throughout the EUV) simultaneously emerge from the focus in a collimated beam embedded in the residual laser beam.

We adapted the polarimeter instrument to accommodate evaporation equipment for in situ deposition of thin films on a substrate, as shown in Fig. 1. We used silicon substrates that were naturally oxidized, with $\mathrm{SiO}_{2}$ layers between 1 and $3 \mathrm{~nm}$ (measured with spectroscopic ellipsometry). At the beginning of a measurement, we placed a substrate in the sample holder and cleaned it with a xenon excimer lamp (Resonance Ltd., Barrie, Ontario, Canada) to minimize hydrocarbon contamination. The system was then pumped to a pressure of $10^{-4}$ Torr with the reflectance chamber and deposition area open to each other. The substrate was then rotated until it pressed against an $\mathrm{O}$ ring attached to a flexible bellows, creating a seal that temporarily isolated the deposition chamber from the reflectance chamber. The deposition chamber was evacuated further to a pressure of $10^{-5}$ Torr.

We prepared the sample by evaporating depleted uranium of purity $>99.7 \%$ horizontally onto the substrates at a distance of $35 \mathrm{~cm}$ using a tungsten basket
(B12B-3X.025W16, R. D. Mathis, Long Beach, California). A shutter covered the substrate during the early phase of evaporation. After approximately $30 \mathrm{~s}$ of evaporation, pressure in the deposition chamber dropped rapidly as the uranium removed molecules in the deposition region, effectively becoming a pump. After the pressure dropped below $10^{-6}$ Torr, the shutter was opened for $10 \mathrm{~s}$. This time frame allowed a deposition of approximately $2-3 \mathrm{~nm}$ of uranium metal (approximately $0.2 \mathrm{~nm} / \mathrm{s}$ ). The sample was deliberately made thin to allow for a more accurate determination of optical constants (see [15]). The sample was rotated back into the measurement chamber within minutes of deposition and without breaking vacuum. The polarization-ratio reflectance measurements were then performed on samples (at room temperature) using the technique described in [15]. After the measurements, the uranium samples were exposed to the atmosphere and the thickness of the oxidized sample was measured with spectroscopic ellipsometry.

We found that our thin films of uranium oxidized completely in less than the time required to vent the chamber and take an ellipsometry measurement $(\sim 5$ min). This result was unexpected because oxidation rates previously reported on bulk samples suggest a slower room-temperature oxidation rate, approximately $0.37 \mathrm{~nm} / h[18,19]$. This rate suggests that we should have been able to see the oxide thickness growing, something we recently observed for copper [7]. Atomic force microscope (AFM) measurements showed that our uranium films had less than $1 \mathrm{~nm}$ rms roughness on a $1 \mu \mathrm{m} \times 1 \mu \mathrm{m}$ scale. We thus hypothesize that (1) very thin films of uranium can oxidize initially more quickly than bulk or thicker films or (2) our samples could have microchasms or voids not visible with the AFM that allow it to oxidize more quickly than a bulk sample.

The film thickness for the completely oxidized uranium was characterized with an accuracy of $\pm 0.3 \mathrm{~nm}$, which was the variation of the thickness across approximately $2 \mathrm{~cm}$ of the sample. The thickness of the unoxidized uranium layer was determined indirectly from the ellipsometric measurement of the

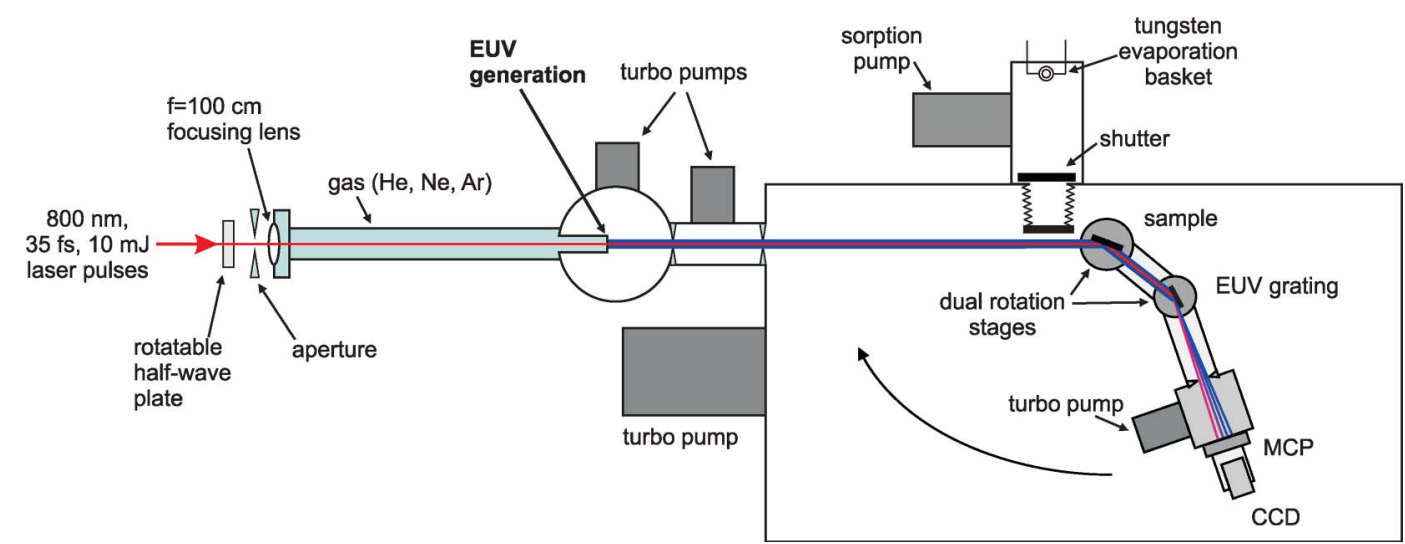

Fig. 1. (Color online) Polarimeter schematic (top view). Laser pulses of $800 \mathrm{~nm}, 35 \mathrm{fs}$, and $10 \mathrm{~mJ}$ are focused into a gas cell to generate harmonics. Thin films are deposited and polarized reflectance measurements are made in situ. 
oxide layer thickness using the ratio of the densities of the metal and the oxide (densities for bulk uranium and $\mathrm{UO}_{2}$ were assumed, 19.1 and $10.96 \mathrm{~g} / \mathrm{cm}^{3}$, respectively). We estimate a thickness accuracy of approximately $\pm 0.7 \mathrm{~nm}$ due to uncertainty in the density and/ or composition of the oxide along with variation across the sample.

To measure the optical constants over a wide range of wavelengths, we took measurements using two diffraction gratings in our system. Changing the grating required us to break vacuum, so the data reported in the next section are from two separate deposition runs. For the short wavelengths, the film thickness was $2.7 \mathrm{~nm}$; for the long wavelengths, the thickness was $3.2 \mathrm{~nm}$.

\section{Ratio Reflectance Data and Optical Constants for Uranium}

Figure 2 shows the ratio reflectance and fit for the uranium film at representative wavelengths of 18.6 and $34.8 \mathrm{~nm}$. Each point represents an average of 400 laser shots at an effective repetition rate of approximately $3 \mathrm{~Hz}$ (because of laser energy discrimination of $\pm 10 \%$ described in [14]). The lines show best-fit curves calculated from the model described in [15] with $n$ and $\kappa$ for the test layer taken as free parameters in a least-squares fitting algorithm (MATLAB nlinfit). The film thickness was held stationary in the fits at the value calculated from the measured thickness of the oxidized layer (as described above). The optical constants for the silicon dioxide layer and silicon substrate were also held stationary.

The uncertainties in film thickness affect the uncertainties for the optical constants. As mentioned previously, the uncertainty in the uranium film thickness was $\pm 0.7 \mathrm{~nm}$. Figure 3 shows how this uncertainty influences the values of optical constants. The solid curve in Fig. 3 shows the constants of uranium that we obtained by using our best estimate for film thickness. The dashed curves show the extent of the change in the optical-constant curve when the thickness was changed by $\pm 0.7 \mathrm{~nm}$.

To estimate our total measurement error we added in quadrature the statistical error due to the fit $(95 \%$ confidence intervals for $n$ or $\kappa$ from the nonlinear regression analysis) and the error due to uncertainty in

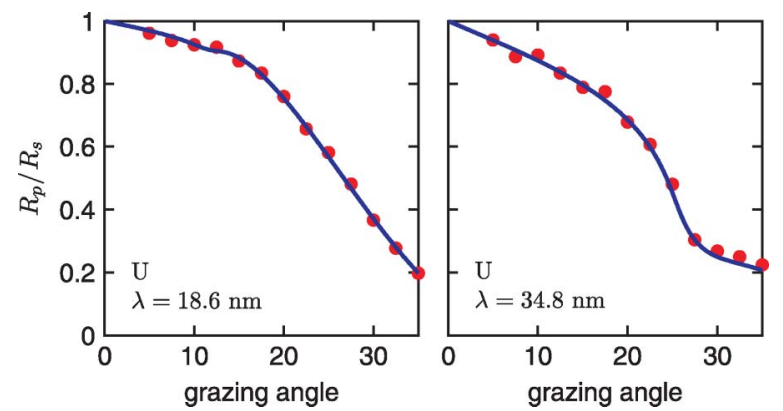

Fig. 2. (Color online) Measured ratio of p-polarized to s-polarized reflectance and fit of uranium at two representative wavelengths: 18.6 and $34.8 \mathrm{~nm}$.

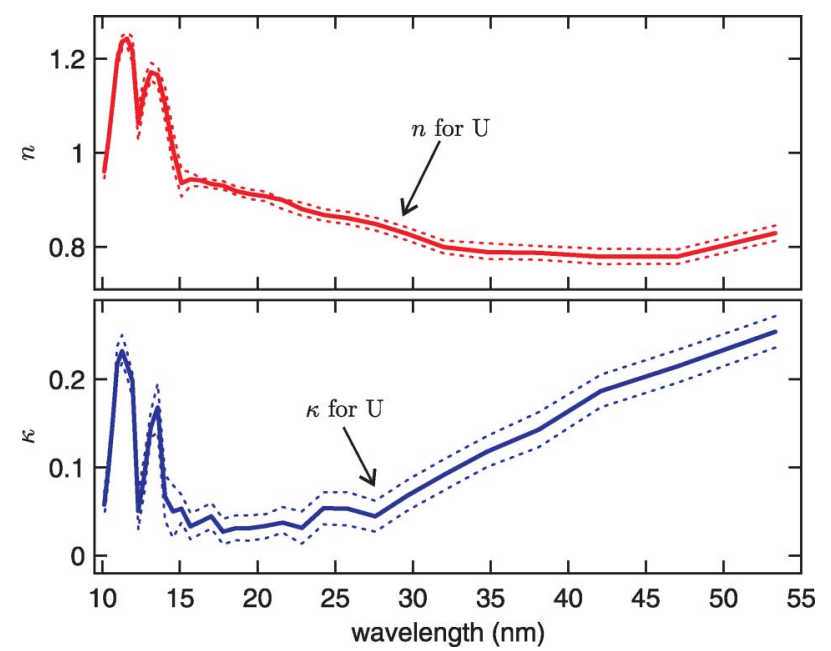

Fig. 3. (Color online) Solid curves: the measured optical constants of uranium; dashed curves: the extent of the change when errors due to uncertainty in thickness are included.

the film thickness. At an example wavelength of $\lambda=$ $13.1 \mathrm{~nm}$ (harmonic order $q=61$ ) our uncertainty of $\pm 1 \mathrm{~nm}$ in film thickness yielded an uncertainty in $n$ of \pm 0.021 and an uncertainty in $\kappa$ of \pm 0.017 . The optical constants for uranium and these error bars are shown in Fig. 4. The optical constants are also listed in tabular form in Table 1 . To test for repeatability, the optical constants of uranium were remeasured completely on a second day with film thicknesses of 2.3 and $2.9 \mathrm{~nm}$. The data sets agree well within our error bars.

\section{Comparison with Previous Measurements and Estimates}

To our knowledge, these data represent the first measurements of the optical constants of uranium in much of the EUV wavelength range. Figure 5 plots our measured optical constants compared with those reported by other sources. Faldt and Nilsson [8]

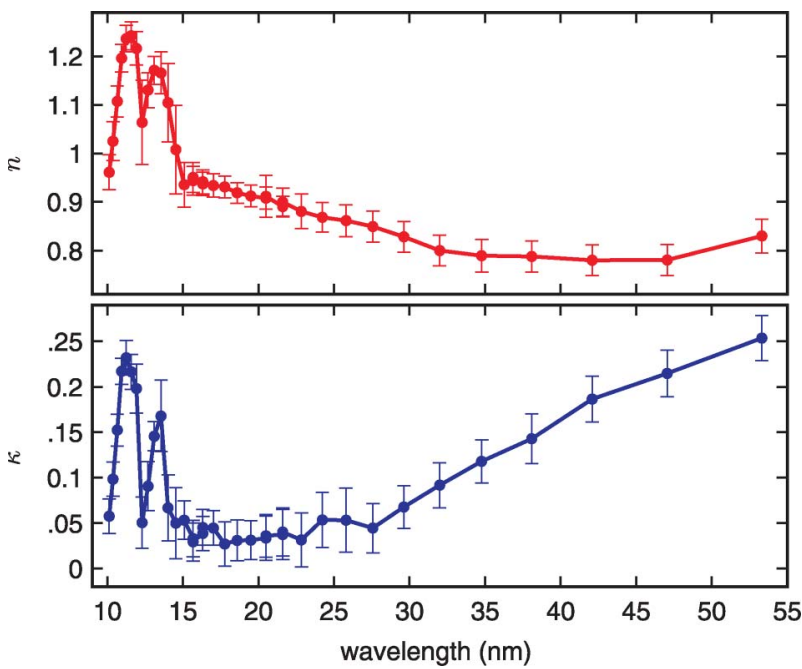

Fig. 4. (Color online) Optical constants of uranium determined using the ratio reflectance technique. Total error bars are also shown. 
Table 1. Optical Constants of Uranium

\begin{tabular}{ccc}
\hline Wavelength & $n$ & $\kappa$ \\
\hline $\mathbf{5 3 . 3}$ & $0.830 \pm 0.035$ & $0.254 \pm 0.025$ \\
$\mathbf{4 7 . 1}$ & $0.780 \pm 0.032$ & $0.215 \pm 0.025$ \\
$\mathbf{4 2 . 1}$ & $0.780 \pm 0.032$ & $0.187 \pm 0.025$ \\
$\mathbf{3 8 . 1}$ & $0.788 \pm 0.032$ & $0.143 \pm 0.027$ \\
$\mathbf{3 4 . 8}$ & $0.789 \pm 0.033$ & $0.118 \pm 0.024$ \\
$\mathbf{3 2 . 0}$ & $0.800 \pm 0.032$ & $0.092 \pm 0.025$ \\
$\mathbf{2 9 . 6}$ & $0.828 \pm 0.032$ & $0.068 \pm 0.024$ \\
$\mathbf{2 7 . 6}$ & $0.849 \pm 0.032$ & $0.045 \pm 0.027$ \\
$\mathbf{2 5 . 8}$ & $0.861 \pm 0.033$ & $0.053 \pm 0.035$ \\
$\mathbf{2 4 . 2}$ & $0.868 \pm 0.030$ & $0.054 \pm 0.030$ \\
$\mathbf{2 2 . 9}$ & $0.880 \pm 0.036$ & $0.032 \pm 0.030$ \\
$\mathbf{2 1 . 6}$ & $0.900 \pm 0.029$ & $0.040 \pm 0.027$ \\
$\mathbf{2 1 . 6}$ & $0.891 \pm 0.021$ & $0.037 \pm 0.028$ \\
$\mathbf{2 0 . 5}$ & $0.911 \pm 0.043$ & $0.036 \pm 0.023$ \\
$\mathbf{2 0 . 5}$ & $0.908 \pm 0.023$ & $0.034 \pm 0.024$ \\
$\mathbf{1 9 . 5}$ & $0.912 \pm 0.023$ & $0.031 \pm 0.021$ \\
$\mathbf{1 8 . 6}$ & $0.919 \pm 0.021$ & $0.031 \pm 0.022$ \\
$\mathbf{1 7 . 8}$ & $0.931 \pm 0.023$ & $0.027 \pm 0.024$ \\
$\mathbf{1 7 . 0}$ & $0.934 \pm 0.024$ & $0.045 \pm 0.019$ \\
$\mathbf{1 6 . 3}$ & $0.941 \pm 0.025$ & $0.045 \pm 0.020$ \\
$\mathbf{1 6 . 3}$ & $0.937 \pm 0.024$ & $0.039 \pm 0.019$ \\
$\mathbf{1 5 . 7}$ & $0.950 \pm 0.031$ & $0.029 \pm 0.021$ \\
$\mathbf{1 5 . 7}$ & $0.944 \pm 0.030$ & $0.033 \pm 0.020$ \\
$\mathbf{1 5 . 1}$ & $0.935 \pm 0.046$ & $0.053 \pm 0.021$ \\
$\mathbf{1 4 . 5}$ & $1.008 \pm 0.091$ & $0.050 \pm 0.039$ \\
$\mathbf{1 4 . 0}$ & $1.104 \pm 0.081$ & $0.067 \pm 0.036$ \\
$\mathbf{1 3 . 6}$ & $1.166 \pm 0.043$ & $0.168 \pm 0.039$ \\
$\mathbf{1 3 . 1}$ & $1.171 \pm 0.029$ & $0.146 \pm 0.016$ \\
$\mathbf{1 2 . 7}$ & $1.130 \pm 0.036$ & $0.091 \pm 0.027$ \\
$\mathbf{1 2 . 3}$ & $1.064 \pm 0.087$ & $0.051 \pm 0.028$ \\
$\mathbf{1 1 . 9}$ & $1.216 \pm 0.035$ & $0.198 \pm 0.027$ \\
$\mathbf{1 1 . 6}$ & $1.242 \pm 0.029$ & $0.217 \pm 0.019$ \\
$\mathbf{1 1 . 3}$ & $1.236 \pm 0.027$ & $0.232 \pm 0.019$ \\
$\mathbf{1 1 . 0}$ & $1.196 \pm 0.028$ & $0.217 \pm 0.015$ \\
$\mathbf{1 0 . 7}$ & $1.107 \pm 0.032$ & $0.152 \pm 0.018$ \\
$\mathbf{1 0 . 4}$ & $1.025 \pm 0.040$ & $0.098 \pm 0.019$ \\
$\mathbf{1 0 . 1}$ & $0.961 \pm 0.036$ & $0.058 \pm 0.019$ \\
\hline & & \\
\hline & & \\
\hline
\end{tabular}

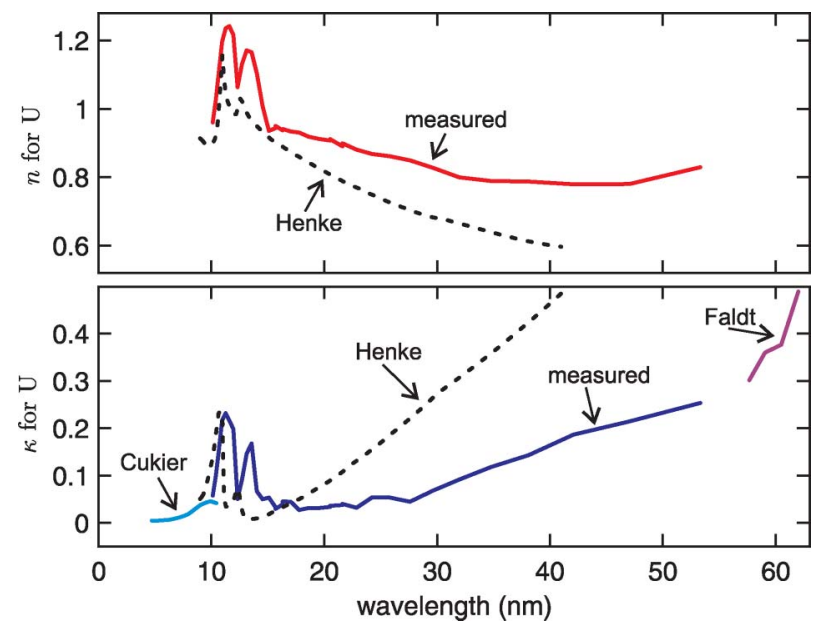

Fig. 5. (Color online) Measured optical constants of uranium compared with constants for uranium measured by Faldt and Nilsson [8] and Cukier et al. [9]. Also shown are optical constants for uranium determined by Henke et al. [10] based on theoretical calculations [11-13].

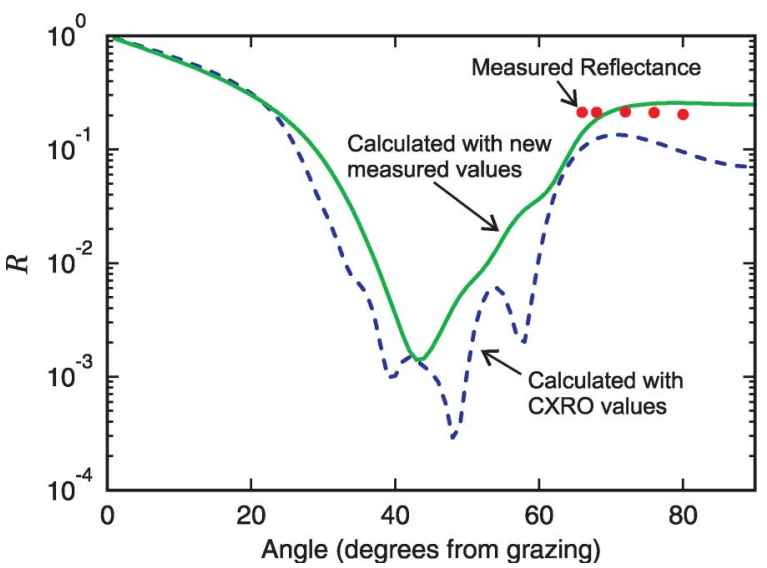

Fig. 6. (Color online) Measured reflectance (dots) for the IMAGE mirror (described in Ref. [1]) compared with the theoretical reflectance computed with our measured optical constants for uranium (solid curve) and computed with the theoretical CXRO constants (dashed curve) at a wavelength of $30.4 \mathrm{~nm}$. Both computed models use the CXRO data for silicon and assume perfectly sharp boundaries between the layers.

measured the optical constants of evaporated uranium in situ from 50 to $2000 \mathrm{~nm}$. The data are consistent with the extension of our data to longer wavelengths. Cukier et al. [9] measured the photoabsorption cross section of uranium from 2.7 to $9.5 \mathrm{~nm}$, preventing oxidation using an aluminum capping layer, and again the data are consistent with our data extended to shorter wavelengths.

Figure 5 also shows the theoretical optical constants for uranium estimated by Henke et al. and cited on the CXRO website $[10,16]$. These constants are based on theoretical calculations of the photoabsorption cross section of uranium [11-13]. Previous to our study, these calculations were the only optical constants for uranium available in this region. Henke and co-workers note that these calculations are only applicable far from absorption thresholds and at wavelengths shorter than $24 \mathrm{~nm}$ [10]. Much of our data was taken at wavelengths longer than $24 \mathrm{~nm}$, and wavelengths shorter than $24 \mathrm{~nm}$ are near an absorption edge $(\sim 11 \mathrm{~nm})$. Therefore, we do not expect these calculations to match the data very well; however, they do confirm the general trend of our data. Notably, the absorption peaks in our measured data are shifted approximately $0.5 \mathrm{~nm}$ to longer wavelengths (lower energy) in comparison with those based on theoretical calculations. A similar resonance shift was also recently noted in the measured constants of thorium and thorium dioxide [20,21].

\section{Conclusions}

We used our high harmonics-based polarimeter to measure the optical constants of uranium in the range from 10 to $47 \mathrm{~nm}$. To the best of our knowledge, this is the first time the constants have been measured for elemental uranium in this wavelength region. We found that measured values differ from theoretical estimates in this range. Our measured optical constants for uranium are consistent with 
the reflectance performance of a multilayer mirror coating created for the IMAGE satellite (see Fig. 6), whereas the previous theoretical estimates are not. This updated knowledge of the optical properties of uranium potentially allows multilayers involving uranium to be designed with improved performance.

This research was supported by the National Science Foundation (NSF) under grant PHY-0457316.

\section{References}

1. B. R. Sandel, A. L. Broadfoot, C. C. Curtis, R. A. King, T. C. Stone, R. H. Hill, J. Chen, O. H. W. Siegmund, R. Raffanti, D. D. Allred, R. S. Turley, and D. L. Gallagher, "The extreme ultraviolet Imager investigation for the IMAGE mission," Space Sci. Rev. 91, 197-242 (2000).

2. G. L. T. Chiu and J. M. Shaw, "Optical lithography: introduction," http://www.research.ibm.com/journal/rd/411/chiu.html (accessed 21 January 2008).

3. A. Hirai, K. Takemoto, K. Nishino, B. Niemann, M. Hettwer, D. Rudolph, E. Anderson, D. Attwood, D. P. Kern, Y. Nakayama, and H. Kihara, "Transmission x-ray microscopy with $50 \mathrm{~nm}$ resolution installed at Ritsumeikan Synchrotron Radiation Center," J. Appl. Phys. 38, 274-278 (1999).

4. B. Sae-Lao and R. Soufli, "Measurements of the refractive index of yttrium in the $50-1300 \mathrm{eV}$ energy region," Appl. Opt. 41, 7309-7316 (2002).

5. R. Soufli and E. Gullikson, "Absolute photoabsorption measurements of molybdenum in the range 60 to $930 \mathrm{eV}$ for optical constant determination," Appl. Opt. 37, 1713-1719 (1998).

6. M. Fernandez-Perea, J. I. Larruquert, J. Aznarez, J. Mendez, M. Vidal-Dasilva, E. Gullikson, A. Aquila, R. Soufli, and J. L. G. Fierro, "Optical constants of electron-beam evaporated boron films in the $6.8-900 \mathrm{eV}$ photon energy range," J. Opt. Soc. Am. A 24, 3800-3807 (2007).

7. N. Brimhall, N. Herrick, D. D. Allred, R. S. Turley, M. Ware, and J. Peatross, "Measured optical constants of copper from $10 \mathrm{~nm}$ to $35 \mathrm{~nm}$," Opt. Express 17, 23873-23879 (2009).

8. A. Faldt and P. O. Nilsson, "Optical properties of uranium in the range 0.6-25 eV," J. Phys. F 10, 2573-2580 (1980).

9. M. Cukier, P. Dhez, F. Wuilleumier, and P. Jaegle, "Photoionization cross section of uranium in the soft x-ray region," Phys. Lett. A 48, 307-308 (1974).
10. B. L. Henke, E. M. Gullikson, and J. C. Davis, "X-ray interactions: photoabsorption, scattering, transmission, and reflection at $E=50-30000 \mathrm{eV}, Z=1-92$," At. Data Nucl. Data Tables 54, 181-342 (1993).

11. G. Wendin and N. Kerr del Grande, "On the photoabsorption cross section of uranium metal," Phys. Scr. 32, 286-290 (1985).

12. J. H. Scofield, "Hartree-Slater subshell photoionization cross sections at 1254 and $1487 \mathrm{eV}$," J. Electron. Spectrosc. Relat. Phenom. 8, 129-137 (1976).

13. E. Plechaty, D. E. Cullen, and R. J. Howerton, "Tables and graphs of photon interaction cross sections from $100 \mathrm{eV}$ to 100 $\mathrm{MeV}$ derived from the LLL evaluated Nuclear Data Library," Lawrence Livermore National Laboratory Report UCRL50400, Vol. 6, Rev. 2 (1978).

14. N. Brimhall, M. Turner, N. Herrick, D. D. Allred, R. S. Turley, M. Ware, and J. Peatross, "Extreme-ultraviolet polarimeter utilizing laser-generated high-order harmonics," Rev. Sci. Instrum. 79, 103108 (2008).

15. N. Brimhall, N. Heilmann, M. Ware, and J. Peatross, "Polarization-ratio reflectance measurements in the extreme ultraviolet," Opt. Lett. 34, 1429-1431 (2009).

16. E. Gullikson, "X-ray interactions with matter," http://henke.lbl .gov/opticalconstants/ (accessed 16 June 2009).

17. R. L. Sandberg, D. D. Allred, S. Lunt, M. K. Urry, and R. S. Turley, "Optical properties and application of uranium-based thin films for the extreme ultraviolet and soft x-ray region," Proc. SPIE 5538, 107-118 (2004).

18. G. W. McGillivray, D. A. Geeson, and R. C. Greenwood, "Studies of the kinetics and mechanism of the oxidation of uranium by dry and moist air: a model for determining the oxidation rate over a wide range of temperatures and water vapor pressures," J. Nucl. Mater. 208, 81-97 (1994).

19. J. M. Haschke, "Corrosion of uranium in air and water vapor: consequences for environmental dispersal," J. Alloys Compd. 278, 149-160 (1998).

20. J. Johnson, D. Allred, R. S. Turley, W. Evans, and R. Sandberg, "Thorium-based thin films as highly reflective mirrors in the EUV," Mater. Res. Soc. Symp. Proc. 893, 207 214 (2006)

21. N. F. Brimhall, A. B. Grigg, R. S. Turley, and D. D. Allred, "Thorium dioxide thin films in the extreme ultraviolet," Proc. SPIE 6317, 631710 (2006). 